\title{
Multiple antibody specificities (gp41, V1V2, and V3) elicited in the phase II multiclade (A, B, C) HIV-1 DNA prime, rAd5 boost vaccine trial
}

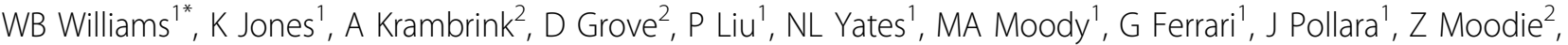 \\ CA Morgan², H Liao' ${ }^{1}$, DC Montefiori ${ }^{1}$, C Ochsenbauer ${ }^{3}$, J Kappes ${ }^{3}$, S Hammer ${ }^{4}$, J Mascola ${ }^{5}$, R Koup ${ }^{5}$, L Corey ${ }^{6}$,



From AIDS Vaccine 2012

Boston, MA, USA. 9-12 September 2012

\section{Background}

The phase II DNA prime, rAd5 boost vaccine (HVTN 204) exhibited sufficient safety and immunogenicity to advance into a phase IIb efficacy trial (HVTN 505) in Ad5 seronegative volunteers in the US. In the RV144 ALVAC prime, A/E gp120 protein boost trial, levels of V1V2 IgG antibodies significantly correlated with decreased risk of infection.

\section{Methods}

Sera from 203 vaccinees receiving VRC-HIVDNA016-00VP DNA (m 0,1,2), and VRC-HIVADV014-00-VP rAd5 (m 6), at two weeks post rAd5 boost, and 208 placebos were examined for binding gp140 and gp120 recombinant proteins, gp41, and an antigen panel of $16 \mathrm{~V} 1 \mathrm{~V} 2$ IgG scaffolds representing clades A, B and C V1V2 sequences. A subset of vaccinees and placebo was screened for binding CD4BS antigens. Monoclonal antibodies were generated from antigen specific memory B cell sorts and tested for binding, neutralization, ADCC and virion capture.

\section{Results}

Clade A and B V1V2 IgG antibodies were elicited in $38.4 \%$ and $19.2 \%$ of vaccinees, respectively. A clonal lineage of 3 gp41 mAbs (CH69, CH70, CH71), and a V3-specific gp120 mAb (CH73) were generated from vaccinees. The gp41 mAbs captured infectious HIV-1 transmitted/ founder viruses, while $\mathrm{CH} 73$ mediated ADCC activity against subtypes $B$ and $C$ infected cells, neutralized subtypes $\mathrm{B}$ and $\mathrm{C}$ tier $1 \mathrm{~A}$ viruses, and bound multiple Envs of subtypes A, B and C.

\section{Conclusion}

The phase II multi-clade DNA prime, rAd5 boost vaccine regimen elicited antibody responses to multiple epitope specificities, including V1V2, V3, and gp41. CH69-CH71 and $\mathrm{CH} 73 \mathrm{mAbs}$ represent the initial human mAbs from HVTN 204 vaccine recipients, and reflect the functional profile of vaccine-elicited antibodies. These data suggest that the HVTN 505 Phase IIb efficacy study using this same vaccine regimen may provide an opportunity to examine a diversity of antibody specificities that have been hypothesized as a correlate of HIV-1 infection risk.

\section{Author details}

${ }^{1}$ Duke Human Vaccine Institute, Duke University Medical Center, Durham, NC, USA. ${ }^{2}$ SCHARP, Fred Hutchinson Cancer Research Center, Seattle, WA, USA. ${ }^{3}$ Department of Medicine, University of Alabama at Birmingham, Birmingham, AL, USA. ${ }^{4}$ Columbia University Medical Center, New York, NY, USA. ${ }^{5}$ Vaccine Research Center, National Institute of Allergy and Infectious, Bethesda, MD, USA. ${ }^{6}$ University of Washington, Seattle, WA, USA. ${ }^{7}$ The Aurum Institute, Johannesburg, South Africa. ${ }^{8}$ Division of Infectious Disease, University of Rochester Medical Center, Rochester, NY, USA.

Published: 13 September 2012

\section{doi:10.1186/1742-4690-9-S2-055}

Cite this article as: Williams et al:: Multiple antibody specificities (gp41, V1V2, and V3) elicited in the phase II multiclade (A, B, C) HIV-1 DNA prime, rAd5 boost vaccine trial. Retrovirology 2012 9(Suppl 2):O55. 